\section{LETTERS TO THE EDITOR}

The thermolabile variant of 5,10-methylenetetrahydrofolate reductase is not associated with Parkinson's disease

Oxidative damage has been suggested as a potential mechanism for both atherosclerosis and neurodegenerative disorders such as Parkinson's disease. ${ }^{1}$ One report has noted a $2 \cdot 5$-fold increased risk of cardiovascular disease among patients with Parkinson's disease, ${ }^{2}$ suggesting that a common genetic variant may contribute to both diseases. One such candidate genetic factor, the ApoE- $\varepsilon 4$ allele, is found at a raised frequency in those with cardiovascular disease and Alzheimer's disease, but was recently shown not to be raised in patients with Parkinson's disease. ${ }^{3}$

Homocysteine is a pro-oxidant that acts via the copper catalysed, oxygen dependent, production of hydrogen peroxide. Moderately raised concentrations of the amino acid homocysteine (mild hyperhomocysteinaemia) confer a twofold to threefold risk of vascular disease. In a significant proportion of cases mild hyperhomocysteinaemia arises from the interaction of the homozygous thermolabile (tt) genotype of 5,10-methylenetetrahydrofolate reductase (MTHFR) with suboptimal folate and B12 nutrition." The tt genotype has also been directly associated with cardiovascular disease. ${ }^{5}$ We therefore determined the prevalence of the tt genotype in patients with Parkinson's disease. Patients with early onset Parkinson's disease $(n=188)$ and matched controls ( $n=184$ ) were selected as described previously. ${ }^{3}$ Cases had initial symptoms before the age of 56 years, and were born after 1924. Controls were age and frequency matched by five-year bands, sex, and urbanrural indicator. Genotyping for the tt allele was performed by polymerase chain reaction and Hinf I digestion of genomic DNA extracted from whole blood. ${ }^{4}$ The tt genotype was present in $9.6 \%(n=18)$ of the patients with Parkinson's disease and $7 \cdot 1 \%$ $(n=13)$ of the controls. In the Parkinson's disease group the heterozygote frequency was $42 \cdot 5 \%(n=80)$ and the non-thermolabile homozygote frequency was $47 \cdot 9 \%$ ( $n=$ $90)$. The corresponding frequencies in the control group were $47.3 \%(n=87)$ and $45 \cdot 6 \%(n=84)$ respectively. There was no significant difference in the frequency distribution of genotypes $\left(\chi^{2}=1 \cdot 26,2 \mathrm{df}, \mathrm{P}=\right.$ 0.53 ) and the odds ratio for the tt genotype was $1.39 \quad(95 \%$ confidence intervals $0 \cdot 63-3 \cdot 12$ )

These results clearly indicate that the tt genotype is not associated with Parkinson's disease and does not explain the finding that patients with Parkinson's disease are at increased risk of vascular disease. The fact that no association is found may indicate that the brain is protected from raised homocysteine concentrations by the preferential accumulation of folate in the CNS, where its concentration is three times that found in serum. Other genetic candidates should be examined for a potential role in oxidative damage, and the importance of common environmental factors such as dietary antioxidants considered

DAWN L HARMON DOROTHY RAMSBOTTOM ALEXANDER S WHITEHEAD Genetics Department, Trinity College, Dublin 2, Ireland YOAV BEN-SHLOMO GEORGE DAVEY-SMITH Department of Social Medicine,
University of Bristol, UK

Correspondence to: Professor AS Whitehead, Genetics Department, Trinity College, Dublin 2, Ireland.

1 Jenner P. Oxidative damage in neurodegenerative disease. Lancet 1994;344:796-8.

2 Ben-Shlomo Y, Marmot MG. Survival and cause of death in a cohort of patients with cause of death in a cohort of patients with parkinsonism: possible clues to aetiology?

3 Whitehead AS, Bertrandy S, Finnan F, Butler A, Davey Smith G, Ben-Shlomo Y Frequency of the apolipoprotein $\mathrm{E} \varepsilon 4$ allele in a case-control study of early onset Parkinson's disease. $f$ Neurol Neurosurg Psychiatry 1996;61:347-51.

4 Harmon DL, Woodside JV, Yarnell JWG, et al. The common thermolabile variant of methylenetetrahydrofolate reductase is a majo determinant of mild hyperhomocysteinaemia. Q7 Med 1996;89:571-7.

5 Gallagher PM, Meleady R, Shields DC, et al. Homocysteine and risk of premature coronary heart disease: evidence for a common gene mutation. Circulation 1996;94:2154-8.

Refsum's disease: long term treatment preserves sensory nerve action potentials and motor function

Refsum's disease is a recessively transmitted disorder characterised by retinitis pigmentosa, polyneuropathy, and cerebellar ataxia associated with tissue storage of phytanic acid. ${ }^{\prime}$ Nerve conduction studies in patients with Refsum's disease show abnormal motor nerve conduction velocity (NCV) with signs of chronic denervation. ${ }^{2}$ Sensory nerve action potentials (SNAPs) are usually absent.2 We present a patient with Refsum's disease with preservation of SNAPs and motor functions after 20 years of continuous therapy with diet restriction and plasmapheresis. Her initial clinical course has been reported. ${ }^{34}$

A 39 year old woman with retinitis pigmentosa and cataracts was admitted in 1976 with a four week history of paraesthesia and weakness. Physical examination showed exfoliative dermatitis, constricted visual fields, right ptosis, and facial diplegia. She had bilateral foot drop and decreased sensation distally. She was areflexic. The peroneal nerves were palpably enlarged at the fibular heads. Protein content of CSF was $210 \mathrm{mg} / \mathrm{dl}$ with normal cell count and glucose. Nerve conduction studies showed reduced motor conduction velocity of the right peroneal nerve $(21.1 \mathrm{~m} / \mathrm{s})$ and of the right median sensory conduction velocity $(34.4 \mathrm{~m} / \mathrm{s})$. Sural nerve biopsy showed an increase in connective tissue and loss of myelin. Serum phytanic acid concentration was $6460 \mu \mathrm{M} / \mathrm{l}$ (normal < $34 \mu \mathrm{M} / \mathrm{l}$ ). She was diagnosed with Refsum's disease and started on a low phytanate diet. She returned within one month with a flaccid tetraparesis and a worsening in nerve conduction studies. A twice weekly plasma exchange programme was instituted. She improved in motor and sensory functions. She could ambulate fully with forearm crutches and a short leg brace in three months. Six months after the initiation of plasma exchange, the phytanic acid concentration declined to $340 \mu \mathrm{M} / \mathrm{l}$.

Subsequently, she was maintained on plasmapheresis once every three to six weeks and low phytanate diet over the next 20 years. The phytanic acid concentration after plasmapheresis remained less than $340 \mu \mathrm{M} / \mathrm{l}$. Repeat nerve conduction studies on eight occasions showed the preservation of the right sural and superficial peroneal SNAPs.

On most recent examination in 1996, she had normal strength, sensation, and reflexes in the upper limbs. She had only slight weakness and reduced sensation in the lower limbs distally. She walked around her house without any assistance but required ankle braces and a cane for longer distances. Nerve conduction studies showed: (1) the median nerves: slightly prolonged distal latency (left/right $4.2 \mathrm{~ms} / 4.0 \mathrm{~ms}$ ); normal $\mathrm{NCV}$ in the forearm segment and minimal F wave latency bilaterally; and no significant drop in the compound muscle action potential (CMAP) amplitudes from the distal (wrist) to the proximal (elbow) stimulation sites on either side. (2) The right peroneal nerves: absent CMAP recording from the extensor digitorum brevis muscle; reduced CMAP amplitude $(1.3 \mathrm{mv})$ recording from the tibialis anterior muscle; and no significant drop in CMAP amplitudes from the distal (the fibula neck) to the proximal (knee) stimulation sites. (3) The right tibial nerve:, absent CMAP recording from the abductor hallucis muscle. (4) Normal sensory nerve conduction in bilateral median and radial, right sural, and right superficial peroneal nerves. Needle examination showed acute and chronic denervation in the distal muscles of the right lower limb including the anterior tibialis and gastrocnemius muscles but no denervation in the proximal muscles of the right lower limb (the vastus lateralis and biceps femoris muscles), the paraspinal muscle in the lumbosacral region, or right first dorsal interosseus muscle. These findings were compatible with a predominantly motor axonal polyneuropathy.

Our patient is unusual as most patients previously reported had different degrees of demyelinating sensorimotor polyneuropathy. The reasons why motor fibres are more affected in our patient are not clear. One possible explanation is that phytanic acid is more soluble in motor nerve membranes than in sensory nerve membranes. The difference in solubility may be caused by the differences in the ganglionside compositions of motor and sensory nerves. ${ }^{5}$ Larger amounts of phytanic acid may accumulate in motor fibres than in sensory fibres and cause more damage.

The beneficial effects of dietary treatment and plasmapheresis in Refsum's disease are established. ${ }^{1}$ The effectiveness of the treatment can be monitored by measuring serum phytanic acid concentrations. However, not all of the clinical findings in Refsum's disease are reversible. ${ }^{1}$ Rapidly developed weakness associated with an acute exacerbation often responds more rapidly and completely to treatment than a gradual onset polyneuropathy. The acute flaccid tetraparesis in our patient responded quickly to plamapheresis. The weakness she has had since is mostly likely due to chronic polyneuropathy.

In conclusion, a predominantly motor axonal polyneuropathy may be seen in Refsum's disease and long term dietary 
treatment and plasmapheresis are effective in preserving sensory nerve potentials and motor function.

\section{JAU-SHIN LOU ROBERT SNYDER ROBERT C GRIGGS Department of Neurology, Department of Neurology,
University of Rochester Medical Center \\ Rochester, NY, USA}

Correspondence to: Dr Jau-Shin Lou, University of Rochester Medical Center, Department of Neurology, Box 673, 601 Elmwood Avenue, Rochester, NY 14642, USA.

1 Skinjedal O, Stokke O, Refsum S. Phytanic acid storage disease. In: Dyck PJ, Thomas Peripheral neuropathy. 3rd ed. Philadelphia: WB Saunders Company, 1993:1149-60.

2 Thomas PK. Classification and electrodiagnosis of hereditary neuropathies. In: Brown WF, Bolton CF, eds. Clinical electromyograWF, Bolton CF, eds. Clinical electromyogra-
phy. 2nd ed. Boston: Butterworthphy. 2nd ed. Boston:

3 Penovich PE, Hollander J, Nusbacher JA Griggs RC, MacPherson J. Note on plasma Griggs RC, MacPherson J. Note on plasma exchange therapy in Ref
Neurol 1978;21:151-3.

4 Pabico RC, Gruebel BJ, McKenna BA, Griggs RC, Hollander J, Nusbacher J, Panner BJ. Renal involvement in Refsum's disease. $\mathrm{Am} \mathscr{f}$ Med 1981;70:1136-43.

5 Ogawa-Goto K, Funamoto $N$, Abe $T$ Nagashima K. Different ceramide compositions of gangliosides between human moto and sensory nerves. $\mathcal{F}$ Neurochem 1990;55: 1486-93.

\section{Contraversive visual tilt illusion associ-} ated with a cerebellar infarction

Visual tilt illusion consists of an abnormal perception of the environment, which seems to be rotated at a variable angle without any change in perception of the other characteristics of the objects. It is sometimes associated with other postural and ocular tilt effects. It can be secondary to disturbances in the peripheral or central vestibular pathways. ${ }^{12}$ Previous reports suggest that cerebellar injuries could also cause it, ${ }^{2}$ but this has not been documented before. We report a case of visual tilt illusion probably associated with an isolated cerebellar lesion, studied with CT and MRI.

A 56 year old man with hypertension and hypercholesterolaemia had a sudden attack of continuous vertigo not related to cephalic movements, with nausea and vomiting and a deviation to the left while walking. When it disappeared, 48 hours later, he complained that he saw objects as if they were tilted to his right by $30^{\circ}$ and they should be rotated anticlockwise-that is, to his left-so as to be perceived as vertical. He had a slight head and body tilt to his left that worsened when he was asked to close his eyes and stand upright. There was no skew deviation or other ocular motor disorders. Fundal photographs were not taken, so that ocular torsion could not be assessed. There were no other alterations on neurological examination. Two weeks later the patient was asymptomatic. Brain CT and MRI showed a right hemispheric cerebellar lesion, suggesting an ischaemic infarction (figure). No brainstem or cortical alterations were found.

Visual tilt illusion has been described in unilateral peripheral vestibular lesions; in brainstem injuries, typically in the Wallenberg syndrome, in other medullary and mesencephalic lesions, and in thalamic and parietoinsular cortex disorders. ${ }^{12}$ The most frequent conditions associated with visual tilt illusion are vascular lesions. ${ }^{1}$ As far as we know, there are no reports on documented isolated cerebellar injuries associated with the illusion.

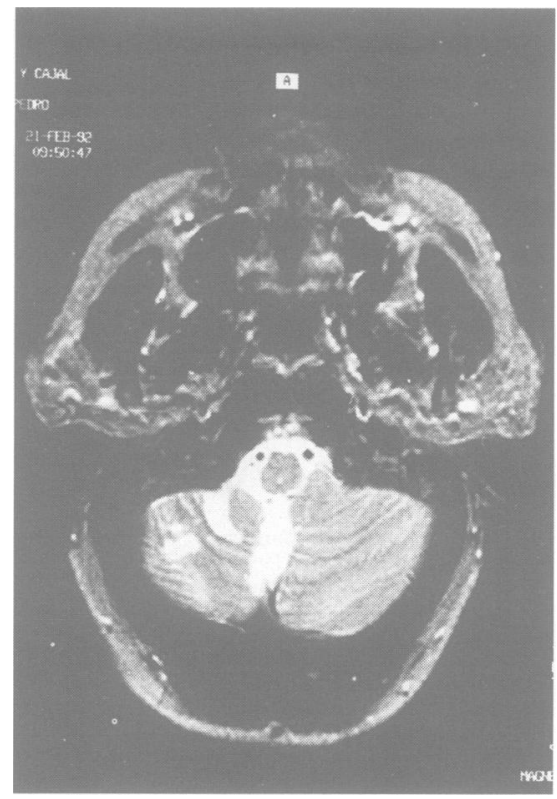

Axial T2 weighted (SE 2000/80) MRI shows an increased signal intensity in the right hemispheric cerebellar region without mass effect, corresponding to an ischaemic infarction in the territory of the posteroinferior cerebellar artery.

Physiologically, the vestibular pathways make contact with the ocular motor system, the spinal cord, and the vestibular cortex, contributing to the stabilisation of posture and perception of verticality and self motion. ${ }^{3-5}$ The tonic bilateral vestibular input builds up the actual central vestibular tone in the three major planes: horizontal or "yaw", sagittal or "pitch", and frontal or "roll". ${ }^{245}$ It seems that central pathways that mediate vestibular function in either of the three planes travel independently of each other, so that a specific lesion could cause a disorder restricted to one of them. ${ }^{24}$ The vestibular tone in the frontal or "roll" plane allows a correct perceptual, ocular, and postural alignment to the "gravitational vertical;" an imbalance in this tone causes a lateral tilt with alteration in perception of verticality, head and body posture, misalignment of the visual axes, or ocular torsion..$^{2-5}$ Patients perceive the surroundings and their body as if they were tilted in the opposite direction to what the CNS erroneously computes as being vertical and try to adjust the visual objects and posture to it. Dieterich and Brandt showed that an alteration in the perceived verticality is not just the sensory consequence of the rotation of the eyes, as they can appear separately and are not proportional in degree. ${ }^{1}$ Furthermore, it is possible that not all the effects of tilt occur in one patient, and the perceptual disorder itself is the most sensitive sign of a vestibular tone imbalance in the frontal plane. ${ }^{15}$ Brainstem structures that mediate the vestibular tone in the "roll" plane include the vestibular nuclei and the intersticial nucleus of Cajal-perhaps the most rostral structure related to the control of vertical and torsional head and eye position. Both are connected by the medial longitudinal fasciculus, which crosses the midline in the pons. ${ }^{24}$ Visual vertical tilt is, then, ipsiversive to peripheral or pontomedullary lesions and contraversive to pontomesencephalic lesions and, in both cases, is usually associated with other tilt effects; in most rostral lesions it may be either ipsiversive or contraversive and is usually isolated. ${ }^{25}$ The role of the vestibular cerebellar structures with respect to the control of subjective verticality is not well known at the moment.

Our patient's clinical findings suggest that he had an inclination of the internal representation of the gravitational vector to his left and he tried to adjust both visual objects and posture to what he perceived as being vertical. It would have been interesting to assess whether there was ocular torsion, to define his clinical setting more exactly, but it makes no difference to interpretation as ocular torsion can be associated or not with perceptual or other tilt effects. ${ }^{1}$ Our patient showed a right hemispheric cerebellar ischaemic lesion, in a territory dependent on the posteroinferior cerebellar artery (PICA), with no mass effect and no brainstem or other alterations on MRI. His perceptual and postural tilt was contraversive to the lesion. It is possible that an additional subtle medullary lesion in the distribution of the PICA, not evident with clinical and imaging studies, produced the tilt effects in this case, because the major infratentorial arteries supply both brainstem and cerebellum and it is very difficult to differentiate the effects of cerebellar and brainstem lesions. ${ }^{2}$ But the tilt should then be ipsiversive, not contraversive, to the hypothetical lesion. Therefore it is not likely that an associated medullary ischaemia could cause the tilt effects in our patient. A mesencephalic injury could cause this clinical picture but there were no other upper brainstem symptoms and MRI was normal at this level. A supratentorial disorder is unlikely because there were no MRI alterations and there were associated postural tilt effects. In this patient, we think that cerebellar dysfunction could be responsible for the tilt effects.

The present report confirms a previously hypothesised role for the cerebellar structures in the control of perception of verticality, ${ }^{2}$ and may contribute to a better knowledge of the pathophysiology and the topographic diagnosis of the central vestibular syndromes.

$M$ BARON JM GOBERNADO MASJUAN

Servicio de Neurología, Hospital Ramón y Cajal, Universidad de Alcalá de Henares, Madrid, España

Correspondence to: $\operatorname{Dr} M$ Barón, Servicio de Neurología, Hospital Ramón y Cajal, Ctra de Colenar, Km 9.100, 28034 Madrid, Spain.

1 Dieterich M, Brandt T. Ocular torsion and tilt of subjective visual vertical are sensitive
brainstem signs. Ann Neurol 1993;33:292-9.

2 Brandt T, Dieterich M. Vestibular syndromes in the roll plane: topographic diagnosis from brainstem to cortex. Ann Neurol 1994;36: 337-47.

3 Rudge P, Bronstein AM. Investigations of disorders of balance. $\mathcal{f}$ Neurol Neurosurg Psychiatry 1995;59:568-78.

4 Leigh RJ, Brandt TH. A re-evaluation of the vestibulo-ocular reflex: new ideas of its purpose, properties, neural substrate, and disorders. Neurology 1993;43:1288-95.

5 Dieterich M, Brandt T. Vestibulo-ocular reflex. Curr Opin Neurol 1995;8:83-8.

Low striatal D2 receptor binding as assessed by [123I]IBZM SPECT in patients with writer's cramp

Writer's cramp is a form of idiopathic focal task specific dystonia. In accord with other studies on idiopathic and symptomatic dystonia, Tempel and Perlmutter suggested the presence of an abnormal striatothalamocortical drive in writer's cramp. ${ }^{1}$ In view of the 